\title{
Subcellular location of antitumor tripeptide-tyroserleutide in human hepatocellular carcinoma cells
}

\author{
XU JIAN ${ }^{1}, \mathrm{ZHENG} \mathrm{FU}^{2}$, YANLING ZHANG $^{3}, \mathrm{XUCHUN} \mathrm{CHE}^{2}, \mathrm{RONG} \mathrm{LU}^{4}$ and ZHI YAO ${ }^{2}$ \\ ${ }^{1}$ Central Laboratory of General Hospital of Tianjin Medical University; Departments of ${ }^{2}$ Immunology, and ${ }^{3}$ Biochemistry, \\ Tianjin Medical University, Tianjin; ${ }^{4}$ Shenzhen Kangzhe Pharmaceutical Co., Ltd., Shenzhen, Guandong, P.R. China
}

Received August 18, 2011; Accepted November 23, 2011

DOI: $10.3892 /$ etm.2011.401

\begin{abstract}
Tyroserleutide (YSL) is a tripeptide compound that exhibits potent antitumor activity in human tumor xenografts and tumor cell lines. However, the target of YSL on which it exerts its antitumor activity has yet to be identified. Therefore, our study aimed to investigate the subcellular location of YSL in BEL-7402 human hepatocellular carcinoma cells. Using methods of fluorescent tracing and confocal colocalization, we provide evidence that when BEL-7402 cells are treated with YSL, YSL is distributed in the cytoplasm and colocalized with the mitochondria of cancer cells. Furthermore, we observed the effect of YSL on the isolated mitochondria. Using fluorescence spectrophotometry to monitor the $\Delta \psi$ collapse of mitochondria isolated from BEL-7402 cells by reversion of the quenching of tetramethylrhodamine methyl ester (TMRM), we found that the isolated mitochondria reversed the quenching of the fluorescence in the solution containing TMRM and YSL. This indicates that YSL decreases the $\Delta \psi$ of the isolated mitochondria. Another photometry method was used to observe the effect on mitochondrial swelling when YSL acted directly on the isolated mitochondria. We reveal that YSL directly causes mitochondrial swelling in $60 \mathrm{~min}$. In conclusion, this study encloses a preliminary facet of the pharmacological target of YSL, and we speculate that YSL may act directly on the mitochondria to exert its antitumor activity.
\end{abstract}

\section{Introduction}

Tyroserleutide (YSL) is a tripeptide compound extracted from the spleen of pigs. It consists of three natural amino acids, L-tyrosine, L-serine and L-leucine. Its chemical structure is shown in Fig. 1. YSL has exhibited potent antitumor activities in human tumor xenografts and tumor cell lines $(1,2)$.

Correspondence to: Dr Xu Jian, Central Laboratory of General Hospital of Tianjin Medical University, No. 154 Anshan Road, Heping, Tianjin 300052, P.R. China

E-mail: jianxu516@hotmail.com

Key words: tyroserleutide, subcellular location, antitumor, target, mitochondria
However, the exact mechanism by which YSL exerts its antitumor activity is not yet fully understood. In our previous study, we observed that YSL induced aptosis and necrosis in BEL-7402 human hepatocellular carcinoma cells in vitro and compromised the organelles of the cancer cells by causing mitochondrial swelling, dissolution and endoplasmic reticulum cisternae expansion $(3,4)$. These observations prompted us to investigate the subcellular location of YSL at the cellular level, with the aim of identifying the pharmacological target implicated in or responsible for YSL-induced apoptosis.

Due to its crucial role in cell apoptosis, the mitochondria have emerged as a novel pharmacological target for anticancer chemotherapy (5,6). A number of anticancer chemotherapeutic drugs that act on mitochondrial targets are under investigation. For example, Bcl-2 ligand HA-14, a small molecule inhibitor of the Bcl-2 family protein, is capable of inducing tumor regression (7). Another mitochondriotoxic lipophilic cation, F16, has been reported to trigger apoptosis and necrosis of carcinoma cells (8). This provides a rationale for investigating the possibility of the mitochondria as the antitumor target of YSL.

In this study, we focus on establishing the subcellular location of YSL in hepatocellular carcinoma cells and the effect of YSL on the isolated mitochondria. Based on these data, we aimed to identify the pharmacological target of YSL and to examine the exact mechanism by which YSL exerts its antitumor activity.

\section{Materials and methods}

Cell culture. BEL-7402, a human hepatocellular carcinoma epithelial cell line (Chinese Medical Academy of Science, Beijing, China), was grown in RPMI-1640 medium (Gibco Invitrogen Corp., Carlsbad, CA, USA) supplemented with 10\% fetal bovine serum (FBS; Hyclone Corp., South Logan, UT, USA), $75 \mu \mathrm{g} / \mathrm{ml}$ penicillin and $100 \mu \mathrm{g} / \mathrm{ml}$ streptomycin at $37^{\circ} \mathrm{C}, 5 \% \mathrm{CO}_{2}$.

YSL fluorescent labeling. YSL (Shenzhen Kangzhe Pharmaceutical Co., Ltd., Shenzhen, China) was reacted with [5-(and-6)-carboxytetramethylrhodamine, succinimidyl ester (TAMRASE; Biotium, Hayward, CA, USA)] at $4^{\circ} \mathrm{C}$ overnight. The bioconjugate was purified by sephadex G-15 chromatographic column and $20 \%$ polyacrylamide gel electrophoresis. Details of the preparation of the fluorescent conjugate are described in our previous study (9). 


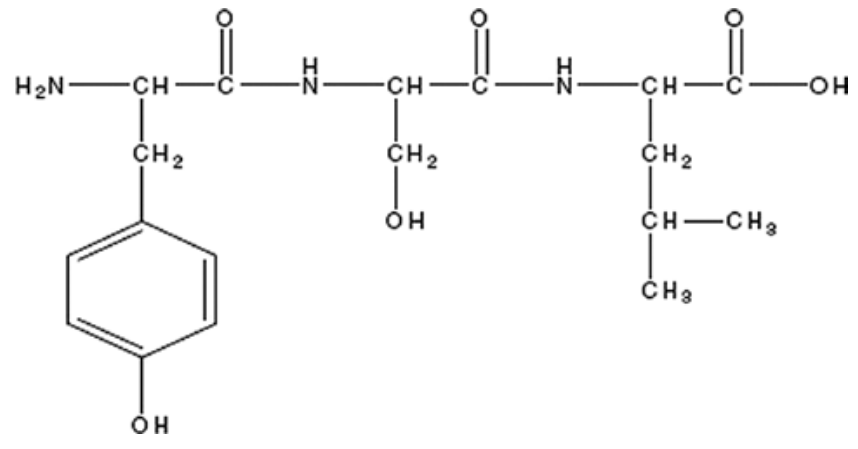

Figure 1. Chemical structure of tyroserleutide.

Confocal microscopy. Human hepatocellular carcinoma cells $\left(1 \times 10^{5} / \mathrm{ml}\right)$ were grown on the cover glass for $24 \mathrm{~h}$, then treated with $26.2 \mu \mathrm{M}$ fluorescent labeled YSL for $1 \mathrm{~h}$. After being washed with D-Hank's solution (Sigma-Aldrich Corp., Shanghai, China), the cells were observed under confocal microscopy (Radiance 2000; Bio-Rad Microscience Corp., Hemel Hempstead, Hertfordshire, UK) using a x60 oil objective lens to examine the subcellular location of YSL. A Bioptech FCS2 chamber (Bioptech Corp., Butler, PA, USA) maintained at $37^{\circ} \mathrm{C}$ was used to examine live cells grown on glass coverslips. To visualize the subcellular compartments, Hoechst 33258 was used (2 $\mu \mathrm{g} / \mathrm{ml}$; Invitrogen Corp.) as a nuclei marker and Mitotracker green FM (200 nM; Life Technologies Corp., Grand Island, NY, USA) as a mitochondrial marker. The lasers that excited the fluorescent analogue of YSL, nuclei marker and mitochondrial marker were blue diode $405 \mathrm{~nm}$, Aron $488 \mathrm{~nm}$ and Green HeNe $543 \mathrm{~nm}$, respectively, and the fluorescent signal was collected using the appropriate filters.

Isolation of cell mitochondria. The BEL-7402 human hepatocellular carcinoma cells $\left(2 \times 10^{7}\right)$ were washed three times with PBS (Sigma-Aldrich Corp.) and centrifuged at 2,500 rpm for $10 \mathrm{~min}$. The supernatant was discarded and the cell pellets were collected for mitochondrial isolation. The mitochondria were isolated using a Mitochondria Isolation kit for Cultured Cells (Pierce Biotechnology, Rockford, IL, USA). The protein concentration of the mitochondria was determined with BCA protein assay reagent (Pierce Biotechnology). The isolated mitochondria were then added to the buffer, which contained $70 \mathrm{mM}$ sucrose, $230 \mathrm{mM}$ mannitol (Kemiou Chemical Reagent Corp., Tianjin, China), 3 mM HEPES (Sigma-Aldrich Corp.), 2 mM Tris-phosphate (Kemiou Chemical Reagent Corp.), $5 \mathrm{mM}$ succinate (Kemiou Chemical Reagent Corp.) and $1 \mu \mathrm{M}$ rotenone (Sigma-Aldrich Corp.).

Mitochondrial potential for isolated mitochondria (10). The mitochondrial potential of isolated mitochondria of BEL-7402 cells was qualitatively assessed using tetramethylrhodamine methyl ester (TMRM; Sigma-Aldrich Corp.) fluorescence intensity. TMRM, a lipophilic cation, accumulates selectively in the mitochondria according to the mitochondrial membrane potential $(\Delta \psi \mathrm{m})(11)$. The high TMRM concentration will quench the mitochondria fluorescence, When mitochondria depolarize, TMRM will be released into the cytosol, and the fluorescence signal increases (12). Mitochondria $(0.35 \mathrm{mg} / \mathrm{ml})$ were added to medium containing TMRM $(1.0 \mu \mathrm{M})$ and
$100 \mu \mathrm{M}$ YSL, and the potential was assessed by reversion of quenching of the fluorescence intensity (excitation/emission $550 / 575 \mathrm{~nm}$ ) using a fluorescent spectrophotometer (F-4500; Hitachi Corp., Japan).

Mitochondrial swelling for isolated mitochondria (13). Mitochondria $(0.5 \mathrm{mg} / \mathrm{ml})$ were incubated with $100 \mu \mathrm{M}$ YSL at $37^{\circ} \mathrm{C}$ for $1 \mathrm{~h}$. The absorbance at $540 \mathrm{~nm}$ was recorded using a spectrophotometer (F-4500; Shimadzu Corp., Kyoto, Japan) every $10 \mathrm{~min}$. Mitochondrial swelling was measured by decrease in absorbance at $540 \mathrm{~nm}$.

\section{Results}

YSL localizes to mitochondria in BEL-7402 hepatocellular carcinoma cells. First, the YSL fluorescent analogue was used to determine the subcellular distribution of YSL in BEL-7402 hepatocellular carcinoma cells. As shown in Fig. 2, YSL primarily located in the cytoplasm was enriched in a definite area. To determine the subcellular compartment in which YSL accumulated, the cells were stained with nuclear and mitochondrial dye. As shown in Fig. 3, YSL was not observed in the nucleus, as demonstrated by a lack of colocalization with cell-permeant DNA stain Hoechst 33258. However, YSL had a high degree of colocalization with mitotracker green FM, a specific mitochondrial probe, indicating that YSL mainly accumulated at the mitochondria of the BEL-7402 cells.

YSL decreases the isolated mitochondrial potential of BEL-7402 cells. Although we found that YSL damages the mitochondria of cancer cells, there is no direct evidence that YSL directly affects the isolated mitochondria. To demonstrate the effect of YSL on isolated mitochondrial potential, we treated the isolated mitochondria with a TMRM mitochondrial potential-sensitive probe to observe the reversion of the fluorescent signal quenching, which represented the decrease in mitochondrial potential. The results indicate that YSL decreases the potential of the isolated mitochondria. When the mitochondria were added to the TMRM solution, the fluorescent signal was quenched. The fluorescence intensity was decreased from 805 to 780 . The fluorescence intensity then rose again and continued to rise. When the mitochondria were added to the TMRM solution for $600 \mathrm{sec}$, the fluorescence intensity rose to 800 ; i.e., $95 \%$ of the original intensity (Fig. 4). These data indicate that the effect of YSL on isolated mitochondria is indicated by the loss of mitochondrial membrane potential, which in this study appears as an increase in TMRM fluorescence intensity.

YSL causes swelling of the isolated mitochondria of BEL-7402 cells. Decrease in the $\Delta \psi \mathrm{m}$ is reminiscent of the opening of the mitochondrial permeability transition (MPT) pore, a key phenomenon in cell death by apoptosis and necrosis. The extensive and prolonged opening of the pore responsible of MPT causes the dissipation of the $\Delta \psi \mathrm{m}$ as well as swelling of the mitochondrial matrix (14). Since YSL induces the disruption of $\Delta \psi \mathrm{m}$, we investigated whether YSL provoked $\Delta \psi \mathrm{m}$ changes dependent on MPT pore opening. The early involvement of the MPT pore opening in YSL may have direct effects on isolated mitochondria. Since the mitochondrial size can be monitored by 


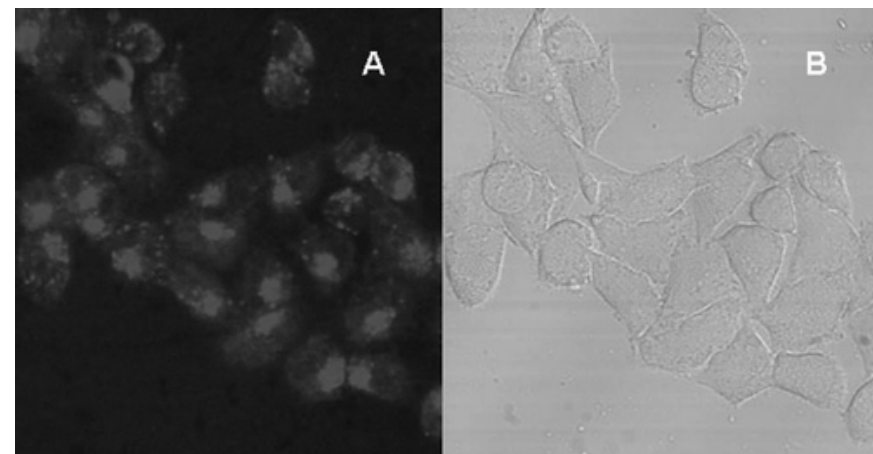

Figure 2. Distribution of YSL in the BEL-7402 human hepatocellular carcinoma cells. BEL-7402 cells were incubated with $26.2 \mu \mathrm{M}$ YSL fluorescent analogue at $37^{\circ} \mathrm{C}$ for $1 \mathrm{~h}$ and then observed under confocal microscopy. The fluorescent signal was collected by laser green HeNe $543 \mathrm{~nm}$ with a x60 oil objective lens. (A) Confocal image; (B) bright field image. YSL, tyroserleutide

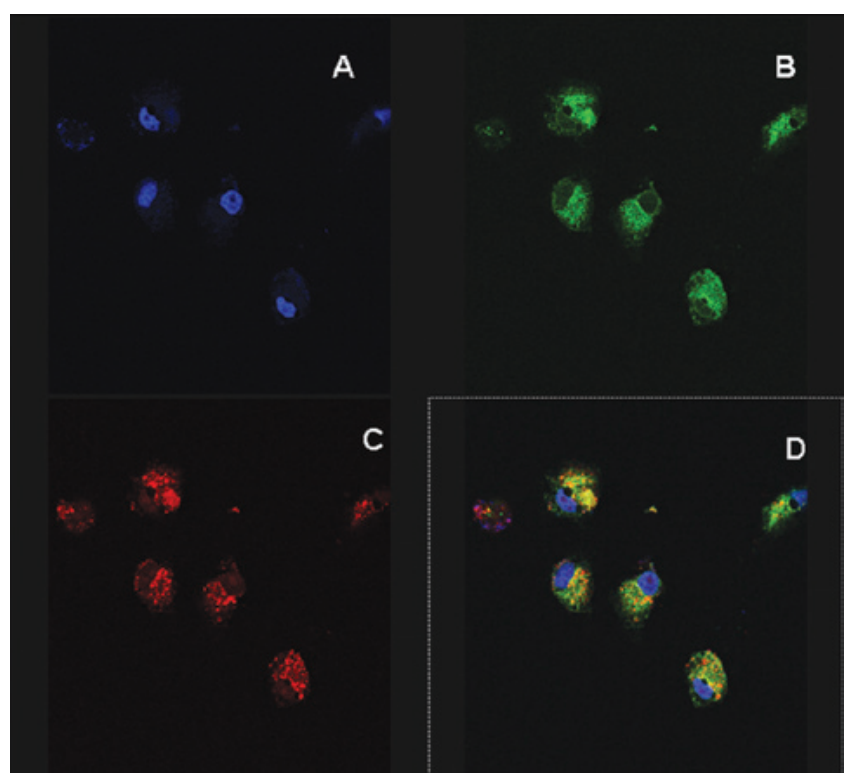

Figure 3. Colocalization of YSL with mitochondria of the BEL-7402 human hepatocellular carcinoma cells under laser scanning confocal microscopy. The fluorescent images were observed by laser scanning confocal microscopy after BEL-7402 cells were incubated with $26.2 \mu \mathrm{M}$ YSL fluorescent analogue, $200 \mathrm{nM}$ Mitotracker Green FM and $2 \mu \mathrm{g} / \mathrm{ml}$ Hoechst 33258 at $37^{\circ} \mathrm{C}$ for $1 \mathrm{~h}$. (A) Blue, Hoechst 33258 stands for nuclei; (B) green, Mitotracker green FM stands for mitochondria; (C) red, YSL fluorescent analogue; (D) merged image. YSL, tyroserleutide.

the changing of the absorbance at $540 \mathrm{~nm}$, we applied spectrophotometry to analyze the mitochondrial size on isolated mitochondria purified from BEL-7402 cancer cells. The results indicated that YSL causes the isolated mitochondria of BEL-7402 cells swelling. After $100 \mu \mathrm{M}$ YSL was incubated with isolated mitochondria for $5 \mathrm{~min}$, the absorbance at $540 \mathrm{~nm}$ was decreased from 1.301 to 1.186 . As the incubation time reached $60 \mathrm{~min}$, the absorbance decreased to 1.091, which indicates that the mitochondria size increased and began to swell (Fig. 5).

\section{Discussion}

This study examined a novel facet of the subcellular distribution of the tripeptide YSL, which possesses a marked

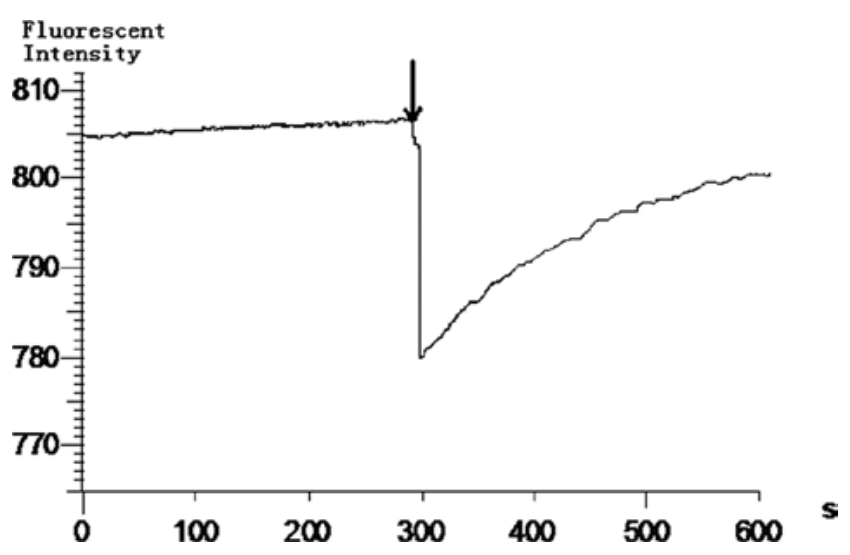

Figure 4. Effect of YSL on mitochondrial potential of the BEL-7402 human hepatocellular carcinoma cells. Isolated mitochondria were incubated with $1.0 \mu \mathrm{M}$ TMRM and $100 \mu \mathrm{M}$ YSL, and the changes in fluorescence intensity following the addition of mitochondria were measured by fluorospectrophotometry to observe the changes of the mitochondrial potential. The arrow indicates the addition of mitochondria. YSL, tyroserleutide; TMRM, tetramethylrhodamine methyl ester.

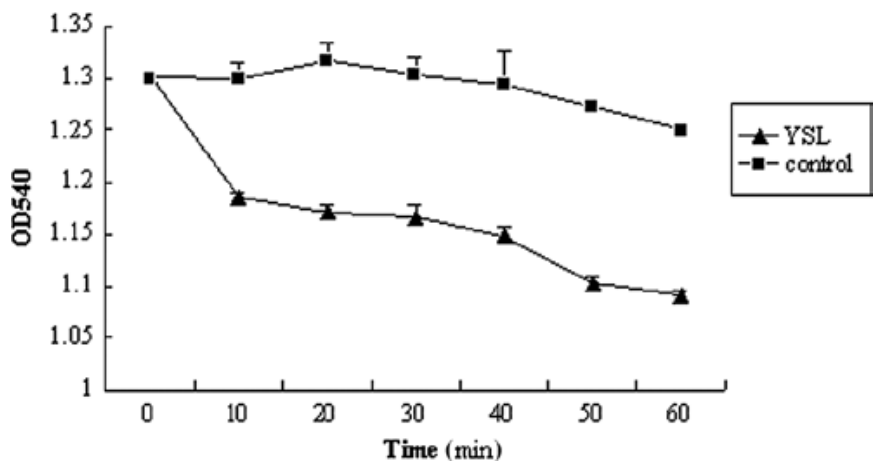

Figure 5. Effect of YSL on mitochondrial swelling of the BEL-7402 human hepatocellular carcinoma cells. Isolated mitochondria of BEL-7402 cells were incubated with $100 \mu \mathrm{M}$ YSL and the absorbance was measured by spectrophotometry at $540 \mathrm{~nm}\left(\mathrm{OD}_{540}\right)$. The intensities were measured every $10 \mathrm{~min}$ and lasted for $60 \mathrm{~min}$ to observe the mitochondrial swelling $(n=5)$. YSL, tyroserleutide.

antitumor effect. In our previous study, we observed that YSL acted as a potent inducer of apoptosis to kill the BEL-7402 hepatocellular carcinoma cells. However, the exact target of the antitumor effect of YSL has yet to be elucidated. YSL belongs to a family of antitumor polypeptides. Due to their marked antitumor effect and lower toxicity, polypeptides with antitumor effects have attracted the attention of investigators studying tumor therapy $(15,16)$. Although the chemical composition of these peptides is similar, their pharmaceutical targets are quite different. Several peptides react with the receptor, which is located at the cell membrane, to stimulate the downstream pathway to kill the cells $(17,18)$. Certain drugs act directly on the intracellular organelles to produce an antitumor effect $(19,20)$. Therefore, variations in the subcellular distribution of the antitumor polypeptide drug in the target cells will determine the various targets of particular antitumor drugs. Thus, we were prompted to investigate the subcellular distribution of YSL to further investigate the target of the antitumor effect.

In our study, we synthesised a fluorescent analogue of YSL using a fluorescence stain [5-(and -6)-carboxytetramethyl- 
rhodamine, succinimidyl ester] to trace YSL in BEL-7402 human hepatocellular carcinoma cells (9). The results revealed that YSL was primarily located at the cytoplasm, whereas the YSL distribution was absent from the cell membrane. These findings indicate that the target of YSL may not be at the membrane of the cells, but in the cancer cells. Since YSL, which consists of tyrosine, serine and leucine, is a water-soluble peptide, it may be passively transported into the tumor cells.

To continue tracing YSL when it enters the tumor cells, we set up a colocalization method. The fluorescent analogue of YSL integrated with other two organelles: A fluorescent probe was used to discover the exact location of YSL in cancer cells under laser scanning confocal microscopy. From the extent of the merging of the YSL fluorescence with the cell organelles, we speculated on the kind of organelle at which YSL was located.

Mounting evidence has revealed that YSL induces the apoptosis of cancer cells affecting various cellular components, including mitochondria, endoplasmic reticulum, histone, protein kinases and phosphates (1-4). Our study identifies the mitochondria as a new pro-apoptotic target of YSL. In the investigation of the location of YSL at the mitochondria, we found that YSL colocalizes with the mitochondria. Indeed, according to our findings, YSL is likely to induce the apoptosis of cancer cells by directly targeting the mitochondria.

As mentioned in the Introduction, the mitochondrion has become a new pharmaceutical target in tumor therapy. Since mitochondria play a significant role in cell apoptosis, drugs that compromise the structure or function of mitochondria will provide opportunities to kill cancer cells if they can be specifically delivered to the tumor site $(21,22)$. In our previous study on YSL-induced cancer cell apoptosis, we found that YSL can cause mitochondrial dysfunction (data not shown). Despite the significance of the mitochondria for the induction of YSL-mediated apoptosis, the results did not explain the mechanism by which YSL activated the mitochondrial pathway. Therefore, we tested this hypothesis on isolated mitochondria. In this study, we found that when YSL acted on isolated mitochondria, it collapsed the $\Delta \psi$ and caused mitochondrial swelling. The dissipation of the $\Delta \psi$ as well as swelling of the mitochondrial matrix were the results of the extensive and prolonged opening of the pore responsible for MPT (23). Therefore, we speculate that the anticancer effect of YSL may involve the opening of the MPT. Further research should be conducted to verify this conclusion.

On theoretical grounds, an agent that directly targets the mitochondria acts on more downstream levels of apoptosis control and may be advantageous for treating cancers, in which such signal transducing systems are interrupted. Therefore, the identification of YSL and its analogue, which directly affects mitochondria, is of substantial clinical interest.

At present, a number of observations have indicated that the antitumor effects of polypeptides involve multiple pathways and multiple targets (24). This study is limited to the subcellular location and the correlation between the direct effect of mitochondria and the antitumor effect of YSL. We will investigate the anticancer mechanism of YSL to clarify the real target of YSL.

In conclusion, we have demonstrated that mitochondria may be the subcellular location of YSL, and YSL directly causes the dissipation of the $\Delta \psi$ as well as mitochondrial swelling. These preliminary findings will contribute to further investigation of the real target of YSL and be beneficial in clinical cancer treatment and the development of the other relevant new drugs.

\section{Acknowledgements}

This study was supported by grants from the National High Technology Research and Development Program of China (2005AA2Z3D40) (863 Program).

\section{References}

1. Yao Z, Lu R, Jia J, et al: The effect of tripeptide tyroserleutide (YSL) on animal models of hepatocarcinoma. Peptides 27: 1167-1172, 2006.

2. Lu R, Jia J, Bao L, et al: Experimental study of the inhibition of human hepatocarcinoma Bel7402 cells by the tripeptide tyroserleutide (YSL). Cancer Chemother Pharmacol 57: 248-256, 2006.

3. Fu Z, Lu R, Li G, et al: Tyroserleutide tripeptide affects calcium homeostasis of human hepatocarcinoma BEL-7402 cells. Sci China C Life Sci 48: 523-530, 2005.

4. Zhao L, Zhao Q, Lu R, et al: Effects of tyroserleutide on gene expression of calmodulin and PI3K in hepatocellular carcinoma. J Cell Biochem 103: 471-478, 2008.

5. Yao J, Jiang Z, Duan W, et al: Involvement of mitochondrial pathway in triptolide-induced cytotoxicity in human normal liver L-02 cells. Biol Pharm Bull 31: 592-597, 2008.

6. Costantini P, Jacotot E, Decaudin D and Kroemer G: Mitochondrion as a novel target of anticancer chemotherapy. J Natl Cancer Inst 92: 1042-1053, 2000.

7. Turner BC, Eves T and Refaeli Y: Small-molecule inhibitors of Bcl-2 family proteins are able to induce tumor regression in a mouse model of pre-B cell acute lymphocytic lymphoma. DNA Cell Biol 27: 133-142, 2008.

8. Fantin VR and Leder P: F16, a mitochondriotoxic compound, triggers apoptosis or necrosis depending on the genetic background of the target carcinoma cells. Cancer Res 1: 329-336, 2004.

9. Jian X, Fu Z, Zhang YL, et al: Synthesis of tyroserleutide fluorescent analogue and its application on the target research of antitumor therapy. Prog Biochem Biophys 35: 1161-1167, 2008.

10. Scaduto RC Jr and Grotyohann LW: Measurement of mitochondrial membrane potential using fluorescent rhodamine derivatives. Biophys J 76: 469-477, 1999.

11. Ehrenberg B, Montana V, Wei MD, Wuskell JP and Loew LM: Membrane potential can be determined in individual cells from the nernstian distribution of cationic dyes. Biophys J 53: 785-794, 1988

12. Korge P, Goldhaber JI and Weiss JN: Phenylarsine oxide induces mitochondrial permeability transition, hypercontracture and cardiac cell death. Am J Physiol Heart Circ Physiol 280: H2203H2213, 2001.

13. Zhao K, Zhao GM, Wu D, Soong Y, Birk AV, Schiller PW and Szeto HH: Cell-permeable peptide antioxidants targeted to inner mitochondrial membrane inhibit mitochondrial swelling, oxidative cell death, and reperfusion injury. J Biol Chem 279: 34682-34690, 2004.

14. Lemasters JJ, Nieminen AL, Qian T, et al: The mitochondrial permeability transition in cell death: a common mechanism in necrosis, apoptosis and autophagy. Biochim Biophys Acta 1366: 177-196, 1998

15. Dias N and Baily C: Drugs targeting mitochondrial function to control tumor cell growth. Biochem Pharmacol 70: 1-12, 2005.

16. Debatin KM, Poncet D and Kroemer G: Chemotherapy: targeting the mitochondrial cell death pathway. Oncogene 21: 8786-8803, 2002.

17. Harsha HC, Jimeno A, Molina H, et al: Activated epidermal growth factor receptor as a novel target in pancreatic cancer therapy. J Proteome Res 7: 4651-4658, 2008.

18. Chene PO, Fuchs JO and Bohn J: A small synthetic peptide which inhibits the p53-hdm2 interaction stimulates the p53 pathway in tumor cell lines. J Mol Biol 299: 245-253, 2000.

19. Papadopoulou LC and Tsiftsoqlou AS: Mitochondrial cytochrome c oxidase as a target site for daunomycin in K-562 cells and heart tissue. Cancer Res 53: 1072-1078, 1993. 
20. Fogal V, Zhang L, Krajewski S and Ruoslahti E: Mitochondrial/ cell-surface protein $\mathrm{p} 32 / \mathrm{gClqR}$ as a molecular target in tumor cells and tumor stroma. Cancer Res 68: 7210-7218, 2008.

21. Rotem R, Heyfets A, Fingrut O, Blickstein D, Shaklai M and Flescher E: Jasmonates: novel anticancer agents acting directly and selectively on human cancer cell mitochondria. Cancer Res 65: 1984-1993, 2005.

22. Lai JC, Tan W, Benimetskaya L, Miller P, Colombini M and Stein CA: A pharmacological target of G3139 in melanoma cells may be the mitochondrial VDAC. PNAS 103: 7494-7499, 2006.
23. Tsujimoto Y and Shimizu S: Role of the mitochondrial membrane permeability transition in cell death. Apoptosis 12: 835-840, 2007.

24. Faivre S, Delbaldo C, Vera K, et al: Safety, pharmacokinetic, and antitumor activity of SU11248, a novel oral multitarget tyrosine kinase inhibitor, in patients with cancer. J Clin Oncol 24: 4-5, 2006. 